\title{
First-in-human phase 1 trial of the safety and immunogenicity of a recombinant adenovirus serotype 5 HVR48 (rAd5HVR48) HIV-1 vaccine
}

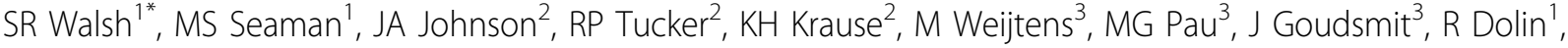 \\ DH Barouch", LR Baden²
}

From AIDS Vaccine 2012

Boston, MA, USA. 9-12 September 2012

\section{Background}

Adenovirus serotype 5 (Ad5) is a potent vector, but widespread seroprevalence may limit its potential use. Replacement of the hexon variable regions (HVR) of Ad5 with the HVR of the less prevalent Ad48 may result in a potent vector which bypasses pre-existing vector immunity.

\section{Methods}

Recombinant Ad5 with seven HVRs derived from Ad48 and expressing the VRC EnvA test antigen (rAd5HVR48. ENVA) was made. 48 healthy volunteers who were seronegative to Ad5, Ad48, HIV-1, and HIV-2 were enrolled in a randomized, double-blind, placebo-controlled, doseescalation phase 1 study. The first three groups of 12 subjects received doses of $10^{9}, 10^{10}$, or $10^{11}$ vp of rAd5HVR48.ENVA vector ( $\mathrm{n}=10$ /group) or placebo $(\mathrm{n}=2 /$ group) at weeks 0,4 , and 24 and the fourth group received a single injection of $10^{10}$ vp or placebo. We performed pre-specified blinded immunogenicity analyses at day 56 and day 196 after the first immunization.

\section{Results}

$31 / 48(65 \%)$ of subjects were female; median age at enrollment was 24 (range: 18-50). Vaccination was generally well tolerated: mild to moderate local and systemic reactogenicity was observed after the initial immunization, more commonly in the highest dose group, but typically resolved within $24 \mathrm{~h}$. No vaccineassociated SAEs occurred. In all four dose groups, 10 subjects per group developed positive EnvA-specific
ELISA titers and EnvA-specific interferon-gamma ELISPOT responses following vaccination. Immune responses were seen two weeks following inoculation in the majority of subjects. Two subjects per group exhibited no vector- or insert-specific immune responses at any timepoint and are presumed placebo recipients.

\section{Conclusion}

The rAd5HVR48 vector is generally safe and immunogenic in humans at all three doses. Immune responses against EnvA could be detected two weeks following the first inoculation. Ad5HVR48 is a promising new chimeric vector to evaluate novel inserts in further clinical trials.

\section{Author details}

${ }^{1}$ Beth Israel Deaconess Medical Center, Boston, MA, USA. ${ }^{2}$ Brigham \& Women's Hospital, Boston, MA, USA. ${ }^{3}$ Crucell, Leiden, Netherlands. ${ }^{4}$ Ragon Institute of MGH, MIT and Harvard, Boston, MA, USA.

Published: 13 September 2012

doi:10.1186/1742-4690-9-S2-O52

Cite this article as: Walsh et al:: First-in-human phase 1 trial of the safety and immunogenicity of a recombinant adenovirus serotype 5 HVR48 (rAd5HVR48) HIV-1 vaccine. Retrovirology 2012 9(Suppl 2):O52.

'Beth Israel Deaconess Medical Center, Boston, MA, USA

Full list of author information is available at the end of the article

(c) 2012 Walsh et al; licensee BioMed Central Ltd. This is an Open Access article distributed under the terms of the Creative Commons 